\title{
Footprint of non-decoupling in chiral phase transition
}

\author{
Tomomi Sato* \\ High Energy Accelerator Research Organization (KEK), Tsukuba 305-0801, Japan \\ School of High Energy Accelerator Science, SOKENDAI (The Graduate University for Advanced \\ Studies), Tsukuba 305-0801, Japan \\ E-mail: tomomisepost.kek.jp

\section{Norikazu Yamada} \\ High Energy Accelerator Research Organization (KEK), Tsukuba 305-0801, Japan \\ School of High Energy Accelerator Science, SOKENDAI (The Graduate University for Advanced \\ Studies), Tsukuba 305-0801, Japan \\ E-mail: norikazu.yamada@kek.jp
}

In the chiral phase transition of massless two flavor QCD, the axial $U(1)$ anomaly may play an important role. We investigate the nature of the transition in the presence of a finite $U_{A}(1)$ breaking by calculating the renormalization group (RG) flow of the effective theory describing massless two flavor QCD at the critical temperature. In a parameter region flowing into an infrared fixed point, we estimate the critical exponents to compare with those in the ordinary $O(4)$ model. It is found that one of the exponents related to the scaling dimension of the leading irrelevant operator is different and that it can be interpreted as an effect of massive degrees of freedom which eventually decouples from the system. Calculations are carried out in the $\varepsilon$ expansion.

The 33rd International Symposium on Lattice Field Theory

14 - 18 July 2015

Kobe International Conference Center, Kobe, Japan*

${ }^{*}$ Speaker. 


\section{Introduction}

Chiral symmetry breaking is one of the most interesting features of QCD. In 1984, Pisarski and Wilczek discussed the order of the chiral phase transition of QCD with massless quarks using linear sigma models (LSMs) and the $\varepsilon$ expansion [1]. They pointed out that the fate of the transition is affected by the $U_{A}(1)$ anomaly in the two flavor case. With an infinitely large breaking of the $U_{A}(1)$ symmetry at $T_{c}$, the phase transition would be described by the $O(4) \mathrm{LSM}$, and hence massless two flavor QCD may undergo a second order phase transition with the $O(4)$ universality class. On the other hand, if the $U_{A}(1)$ symmetry is effectively and fully restored at $T_{c}$, the LSM with $U(2) \times U(2)$ symmetry should be analyzed, and its fate is under active investigation $[2,3,4,5,6,7,8,9,10$, $11,12,13]$. Importantly, the nature of the transition could have an impact on the axion abundance through the temperature dependence of the topological susceptibility [14].

In Ref. [8], we investigated the case where a finite $U_{A}(1)$ breaking remains at $T_{c}$ and tried to see the impact of the breaking on the phase transition by applying the $\varepsilon$-expansion to the $U(2) \times U(2)$ LSM with a finite $U_{A}(1)$ breaking, which we call $U_{A}(1)$ broken model. The findings are briefly summarized in sec. 2. In this work, we examine, in a parameter region flowing into the infrared fixed point (IRFP), the critical exponents of the $U_{A}(1)$ broken model and the equivalence to the $O(4)$ model in the IR limit. We then conclude that the second order phase transition in the $U_{A}(1)$ broken model belongs to the $O(4)$ universality class, but the scaling dimension of the leading irrelevant operator is different from that in the ordinary $O(4)$ LSM.

\section{2. $U_{A}(1)$ broken model}

The $U_{A}(1)$ broken model is the effective theory describing the chiral phase transition of massless two flavor QCD in the presence of a finite $U_{A}(1)$ breaking. The model contains two species of four component real scalars, $\phi_{a}$ and $\chi_{a}(a=0,1,2,3)$, as

$$
\begin{aligned}
\mathscr{L}_{U_{A}(1) \mathrm{br}}= & (1+w) \frac{1}{2}\left(\partial_{\mu} \phi_{a}\right)^{2}+\frac{1}{2}\left(m^{2}-\frac{c_{A}}{2}\right) \phi_{a}{ }^{2}+(1-w) \frac{1}{2}\left(\partial_{\mu} \chi_{a}\right)^{2}+\frac{1}{2}\left(m^{2}+\frac{c_{A}}{2}\right) \chi_{a}{ }^{2} \\
& +\frac{\pi^{2}}{3}\left[\lambda\left(\phi_{a}{ }^{2}\right)^{2}+(\lambda-2 x)\left(\chi_{a}{ }^{2}\right)^{2}+2\left(\lambda+g_{2}-z\right) \phi_{a}{ }^{2} \chi_{b}{ }^{2}-2 g_{2}\left(\phi_{a} \chi_{a}\right)^{2}\right] .
\end{aligned}
$$

For simplicity, we take $w=0$ in the following. $m^{2}$ is used to bring the system into the critical temperature. Then, there remain five free parameters, $\lambda, g_{2}, c_{A}, x, z$. This model has $S U_{L}(2) \times$ $S U_{R}(2) \times Z_{2}$ symmetry. When one takes $x, z$ and $c_{A}$ to zero, $Z_{2}$ is enhanced to $U_{A}(1)$ symmetry, and the model becomes the $U(2) \times U(2)$ LSM. The masses of $\phi_{a}$ and $\chi_{a}$ are split by the $U_{A}(1)$ breaking coefficient $c_{A}$ as

$$
m_{\phi}^{2}=m^{2}-\frac{c_{A}}{2}, m_{\chi}^{2}=m^{2}+\frac{c_{A}}{2} .
$$

Since we are interested in the renormalization group (RG) flow at $T_{c}, m^{2}$ is tuned to realize $m_{\phi}^{2}=0$. Then $m_{\chi}^{2}=c_{A}$. Throughout this calculation, we assume $c_{A}>0$ and $d=4-\varepsilon$. In order to see whether the continuous phase transition is possible, we calculated the RG flow of this model with various initial values. It is naively expected that the massive fields, $\chi$, decouple from the system as 
the scale approaches the IR limit and that the model eventually reduces to the $O(4)$ LSM,

$$
\mathscr{L}_{O(4)}=\frac{1}{2}\left(\partial_{\mu} \phi_{a}\right)^{2}+\lambda_{O(4)}\left(\phi_{a}^{2}\right)^{2} .
$$

To monitor how the decoupling proceeds, the mass dependent renormalization scheme was chosen in the calculation of the $\beta$ functions of the dimensionless couplings, $\hat{\lambda}=\lambda \mu^{-\varepsilon}, \hat{g}_{2}=g_{2} \mu^{-\varepsilon}$, $\hat{x}=x \mu^{-\varepsilon}, \hat{z}=z \mu^{-\varepsilon}$.

It turned out that there is a parameter region where $\hat{\lambda}(\mu)$ approaches its IRFP as

$$
\lim _{\mu \rightarrow 0} \hat{\lambda}(\mu)=\frac{\varepsilon}{2}+a \hat{\mu}^{2-\frac{5}{3} \varepsilon}
$$

where $a$ is a constant. Interestingly, $\hat{\lambda}_{\mathrm{IRFP}}=\varepsilon / 2$ agrees with the Wilson-Fisher FP in the $O(4)$ LSM. However, at the same time, the other couplings turned out to diverge as $\mu \rightarrow 0$ as

$$
\begin{aligned}
& \hat{g}_{2}(\mu \rightarrow 0) \propto \hat{\mu}^{-\frac{5}{6} \varepsilon}, \\
& \hat{x}(\mu \rightarrow 0) \propto \hat{g}_{2}^{2}(\mu \rightarrow 0), \\
& \hat{z}(\mu \rightarrow 0) \propto \hat{g}_{2}(\mu \rightarrow 0) .
\end{aligned}
$$

Furthermore, it also turned out that the second term in eq. (2.4) is different from the $O(4)$ LSM, where

$$
\hat{\lambda}_{O(4)}(\mu \rightarrow 0)=\frac{\varepsilon}{2}+b \hat{\mu}^{\varepsilon},
$$

where $b$ is a constant. The power of $\mu$ is related to the approaching rate to the IRFP, and gives the $R G$ dimension of the leading irrelevant operator in a given theory. Following the literature, we call it $\omega$. It is important to note that $\omega$ characterizes the sub-leading behavior of the critical phenomena and is measurable. For instance, let $\chi(t, h)$ be a magnetic susceptibility ${ }^{1}, t=\left(T-T_{c}\right) / T$ the reduced temperature, and $h$ a external magnetic field, $\omega$ appears like [17]

$$
\chi(t, h=0) \sim t^{-v(2-\eta)}\left(1+C_{\chi} t^{v \omega}+\ldots\right) .
$$

For the two models, the $U_{A}(1)$ broken and $O(4)$ models, they are given by

$$
\omega_{U_{A}(1) \text { br }}=2-\frac{5}{3} \varepsilon, \quad \omega_{O(4)}=\varepsilon,
$$

respectively. It should be noted that this difference arises only when $\varepsilon \geq 3 / 4$, otherwise $\omega_{U_{A}(1) \text { br }}=$ $\varepsilon$. The question to be addressed below is whether the $U_{A}(1)$ broken model undergoes second order phase transition, and if it does, to which universality class it belongs. In the following sections, we examine the IR limit of the the $U_{A}(1)$ broken model.

\section{Correlation functions in the IR limit}

In order to see whether the $U_{A}(1)$ broken model in the attractive basin is equivalent to the $O(4)$ LSM in the IR limit, we compare 1PI $N$-point correlation functions of $\phi$, i.e. $\Gamma_{U_{A}(1) \mathrm{br}}^{(N)}, \Gamma_{O(4)}^{(N)}$, in the

\footnotetext{
${ }^{1}$ Note that it has nothing to do with $\chi$ fields in eq. (2.1).
} 
IR limit at the one loop level. If they agree for arbitrary $N$, we could say that the two models are equivalent in the IR limit and hence the $U_{A}(1)$ broken model undergoes the same phase transition with the $O(4)$ model.

Since the agreements of two and four point functions were confirmed by the explicit calculation in [8], here we focus on $\Gamma^{(N)}$ with $N \geqq 6$. Because of the absence of odd-point interactions, only the case with even $N$ are considered.

After the renormalization group improvement, the 1PI $N$-point correlation functions with an external momentum $P$ are found to be

$$
\begin{gathered}
\Gamma_{U_{A}(1) \mathrm{br}}^{(N)} \sim \bar{\lambda}^{N / 2}(P)\left(\frac{1}{P^{2}}\right)^{\frac{N-d}{2}}+\bar{g}_{2}^{N / 2}(P)\left(\frac{1}{P^{2}+c_{A}}\right)^{\frac{N-d}{2}} \\
\stackrel{P \rightarrow 0}{\longrightarrow} P^{-N+d}\left\{\bar{\lambda}^{N / 2}(P)+\bar{g}_{2}^{N / 2}(P)\left(\frac{P^{2}}{c_{A}}\right)^{\frac{N-d}{2}}\right\}
\end{gathered}
$$

in the leading order of the $\varepsilon$ expansion for the $U_{A}(1)$ broken model, where $\bar{\lambda}(P=\mu)=\hat{\lambda}(\mu)$ and $\tilde{g}_{2}(P=\mu)=\hat{g}_{2}(\mu)$ evolve following their respective RG equations. According to those, as $P \rightarrow 0$ they show the IR asymptotic behaviors,

$$
\begin{aligned}
& \lim _{P \rightarrow 0} \bar{\lambda}(P)=\frac{\varepsilon}{2}+a\left(\frac{P}{\mu}\right)^{2-\frac{5}{3} \varepsilon}, \\
& \lim _{P \rightarrow 0} \bar{g}_{2}(P)=b\left(\frac{P}{\mu}\right)^{-\frac{5}{6} \varepsilon} .
\end{aligned}
$$

Substituting these behaviors and $d=4-\varepsilon$ into eq. (3.1), we obtain for the second term

$$
\hat{g}_{2}^{N / 2}(P)\left(\frac{P^{2}}{c_{A}}\right)^{\frac{N-d}{2}} \stackrel{P \rightarrow 0}{\longrightarrow} P^{\left(1-\frac{5}{12} \varepsilon\right) N-4+\varepsilon} .
$$

Thus, for $N>(4-\varepsilon) /(1-5 \varepsilon / 12) \stackrel{\varepsilon \rightarrow 1}{\longrightarrow} 36 / 7$, this term vanishes as $P \rightarrow 0$ while $\bar{\lambda} \rightarrow \varepsilon / 2$.

On the other hand, $\Gamma^{(N)}$ in the $O(4)$ LSM behaves in the IR region as

$$
\Gamma_{O(4)}^{(N)} \stackrel{P \rightarrow 0}{\longrightarrow} P^{-N+d} \bar{\lambda}_{O(4)}^{N / 2}(P)
$$

Therefore, both $\Gamma_{U_{A}(1) \text { br }}^{(N)}$ and $\Gamma_{O(4)}^{(N)}$ vanish for $N \geqq 6$. Combining with the fact that the same happens to $\Gamma^{(2)}$ and $\Gamma^{(4)}$, we eventually obtain

$$
\lim _{P \rightarrow 0} \Gamma_{U_{A}(1) \mathrm{br}}^{(N)}(P)=\lim _{P \rightarrow 0} \Gamma_{O(4)}^{(N)}(P)
$$

for any $N$ at the one loop level. Because of this agreement, we conclude that two models are equivalent at very low energy scale and hence seem to undergo the same phase transition although some of the couplings in the $U_{A}(1)$ broken model diverge. 


\section{Critical exponents}

Next, we calculate the critical exponents $v$ and $\eta$ in the $U_{A}(1)$ broken model to compare with those in the $O(4)$ LSM. The exponent $v$ characterizes the divergence of a correlation length $\xi$ as

$$
\xi \sim\left(\frac{\left|T-T_{c}\right|}{T_{c}}\right)^{v}
$$

and $\eta$ appears in the correlation function as

$$
\left\langle\phi_{a}(x) \phi_{a}(0)\right\rangle \sim|x|^{-d+2-\eta} .
$$

They are related to the anomalous dimensions of $\phi$ and $\phi^{2}$ respectively as

$$
v=\frac{1}{2-\gamma_{\phi^{2}}^{*}}, \quad \eta=2 \gamma_{\phi}^{*},
$$

where the superscript ${ }^{*}$ denotes the anomalous dimension in the IR limit.

At the leading order of the $\varepsilon$ expansion, the anomalous dimension of $\phi$ is zero in the both models. Hence

$$
\eta_{U_{A}(1) \mathrm{br}}=\eta_{O(4)}=0
$$

This is because of the absence of the wave function renormalization in the $\phi^{4}$ like theory at the leading order of the $\varepsilon$ expansion. Thus, the agreement of $\eta$ seems somewhat trivial.

In the $O(4)$ LSM, the anomalous dimension of $\phi_{a}^{2}$ is obtained as [19]

$$
\gamma_{\phi^{2}, O(4)}=\hat{\lambda}_{O(4)}(\mu)
$$

in the leading order of the $\varepsilon$ expansion. On the other hand, $\phi_{a}^{2}$ mixes with $\chi_{a}^{2}$ in the $U_{A}(1)$ broken model as

$$
\left(\begin{array}{l}
{\left[\phi_{a}^{2}\right]} \\
{\left[\chi_{a}^{2}\right]}
\end{array}\right)=Z_{m^{2}}\left(\begin{array}{c}
\phi_{a}^{2} \\
\chi_{a}^{2}
\end{array}\right),
$$

where $\left[\phi_{a}^{2}\right]$ and $\left[\chi_{a}^{2}\right]$ represent the renormalized operators. By diagonalizing $Z_{m^{2}}$, we obtain

$$
P^{-1} Z_{m^{2}} P=\operatorname{diag}\left\{Z_{+}, Z_{-}\right\},
$$

and then the eigenvectors are found to be

$$
\left(\begin{array}{c}
\Phi_{+}^{2} \\
\Phi_{-}^{2}
\end{array}\right)=P^{-1}\left(\begin{array}{c}
\phi_{a}^{2} \\
\chi_{a}^{2}
\end{array}\right) .
$$

Noting that the mixing matrix $P$ becomes the identity matrix in the IR limit, and defining

$$
\gamma_{ \pm}=-\mu \frac{d}{d \mu} \log Z_{ \pm}
$$


$\gamma_{\phi^{2}, U_{A}(1) b r}^{*}$ is obtained from

$$
\gamma_{\phi^{2}, U_{A}(1) \mathrm{br}}^{*}=\lim _{\mu \rightarrow 0} \gamma_{+}=\lim _{\mu \rightarrow 0} \hat{\lambda}(\mu)
$$

As seen above, $\hat{\lambda}$ in the two models falls into the same IRFP. Therefore, the exponent $v$ in the two models agree, too, and take

$$
v_{U_{A}(1) \mathrm{br}}=v_{O(4)}=\frac{1}{2}+\frac{\varepsilon}{8}+\mathscr{O}\left(\varepsilon^{2}\right) .
$$

Using the scaling relations, $\alpha=2-d v$ and $\beta=v(d-2+\eta) / 2$, and so on, all other critical exponents agree between the $U_{A}(1)$ broken and the $O(4)$ models.

On the other hand, we have seen a discrepancy in the exponent $\omega$. It is then interesting to consider the low energy effective theory of the $U_{A}(1)$ broken model, where $\chi$ fields are integrated out. One possibility for the resulting effective theory is the $O(4)$ LSM including $O(4)$ symmetric irrelevant operators, the most relevant one of which would lead to $\omega_{U_{A}(1) \mathrm{br}}$ different from $\omega_{O(4)}$. As long as we have glanced, we could not find such a operator and hence it is unlikely to be the case. Another possibility is the $O(4)$ LSM including irrelevant operators not symmetric under $O(4)$ rotation. Although some related arguments are found in [18], we are not very sure how those arguments fit our case. At any rate, it is clearly interesting to understand this point, especially whether we can identify the footprint of the existence of the massive fields from the sub-leading behavior of the critical phenomena. To check this, numerical simulations are ongoing.

\section{Summary}

We investigated the low energy behavior of the $U_{A}(1)$ broken model by applying the $\varepsilon$-expansion analysis. Starting from the inside of the attractive basin of the $U_{A}(1)$ broken model, we found that the massive fields $\chi$ decouple from the system in the IR limit by seeing that the correlation functions of $\phi$ in the $U_{A}(1)$ broken model converge to those of $O(4)$ model in the IR limit. Hence, it seems that the $U_{A}(1)$ broken model will end up with second order phase transition because the $O(4)$ does. The coincidence of $v$ and $\eta$ and hence all the critical exponents gives further support. On the other hand, we also found that, at the same time, $\chi$ can affect the sub-leading behavior of the critical phenomena by observing $\omega_{U_{A}(1) \text { br }}$ different from $\omega_{O(4)}$. If the decoupling theorem $[15,16]$ states that heavy particles can not contribute to any observables in the IR limit, the decoupling theorem still holds. However, it is interesting to understand whether the massive fields contribute to the low energy observables, e.g. $\omega$, before reaching the IR limit.

This work is in part supported by JSPS KAKENHI Grant-inAid for Scientific Research (B) (No. $15 \mathrm{H} 03669[\mathrm{NY}])$.

\section{References}

[1] R. D. Pisarski and F. Wilczek, "Remarks on the Chiral Phase Transition in Chromodynamics," Phys. Rev. D 29, 338 (1984). 
[2] S. Aoki, H. Fukaya and Y. Taniguchi, "Chiral symmetry restoration, eigenvalue density of Dirac operator and axial U(1) anomaly at finite temperature," Phys. Rev. D 86 (2012) 114512 [arXiv:1209.2061 [hep-lat]].

[3] S. Ejiri and N. Yamada, "End Point of a First-Order Phase Transition in Many-Flavor Lattice QCD at Finite Temperature and Density,” Phys. Rev. Lett. 110 (2013) no.17, 172001 doi:10.1103/PhysRevLett.110.172001 [arXiv:1212.5899 [hep-lat]].

[4] G. Cossu, S. Aoki, H. Fukaya, S. Hashimoto, T. Kaneko, H. Matsufuru and J. -I. Noaki, "Finite temperature study of the axial U(1) symmetry on the lattice with overlap fermion formulation," Phys. Rev. D 87 (2013) 114514 [arXiv:1304.6145 [hep-lat]].

[5] M. I. Buchoff, M. Cheng, N. H. Christ, H. -T. Ding, C. Jung, F. Karsch, R. D. Mawhinney and S. Mukherjee et al., "The QCD chiral transition, $U_{A}(1)$ symmetry and the Dirac spectrum using domain wall fermions," arXiv:1309.4149 [hep-lat].

[6] A. Pelissetto and E. Vicari, "Relevance of the axial anomaly at the finite-temperature chiral transition in QCD,” Phys. Rev. D 88 (2013) 10, 105018 [arXiv:1309.5446 [hep-lat]].

[7] Y. Nakayama and T. Ohtsuki, "Bootstrapping phase transitions in QCD and frustrated spin systems," Phys. Rev. D 91 (2015) 2, 021901 [arXiv:1407.6195 [hep-th]].

[8] T. Sato and N. Yamada, "Linking $U(2) \times U(2)$ to $O(4)$ model via decoupling," Phys. Rev. D 91 (2015) 3, 034025 [arXiv:1412.8026 [hep-lat]].

[9] T. Sato and N. Yamada, "Renormalization group flow of linear sigma model with $U_{A}(1)$ anomaly," PoS LATTICE 2014 (2015) 191 [arXiv:1501.06684 [hep-lat]].

[10] G. Cossu, S. Hashimoto, H. Fukaya, A. Tomiya, T. Kaneko, J. -I. Noaki, these proceedings.

[11] A. Tomiya, G. Cossu, H. Fukaya, S. Hashimoto, T. Kaneko, J. -I. Noaki, these proceedings.

[12] S. Sharma, V. Dick, F. Karsch, E. Laermann, S. Mukherjee, these proceedings.

[13] K. Kamikado, these proceedings.

[14] R. Kitano and N. Yamada, “Topology in QCD and the axion abundance," JHEP 1510, 136 (2015) doi:10.1007/JHEP10(2015)136 [arXiv:1506.00370 [hep-ph]].

[15] K. Symanzik, "Infrared singularities and small distance behavior analysis," Commun. Math. Phys. 34, 7 (1973).

[16] T. Appelquist and J. Carazzone, "Infrared Singularities and Massive Fields,” Phys. Rev. D 11, 2856 (1975).

[17] E. Brezin, J-C. Le Guillous, and J. Zinn-Justin, Phys. Rev. B 8, 5330 (1973).

[18] M. Hasenbusch, E. Vicari, Phys. Rev. B 84, 125136 (2011).

[19] E. Brezin, J. C. Le Guillou and J. Zinn-Justin, "Wilson's theory of critical phenomena and callan-symanzik equations in 4-epsilon dimensions," Phys. Rev. D 8 (1973) 434. 